\title{
Clinicopathologial Features of Gastric Hepatoid Adenocarcinoma
}

\author{
Cheng-Yu Lin ${ }^{1}$, Huei-Chung Yeh², Chen-Ming Hsu ${ }^{1,3}$, Wey-Ran Lin ${ }^{1,3}$, Cheng-Tang Chiu ${ }^{1,3}$
}

Background: Gastric hepatoid adenocarcinoma (GHA), a rare type of primary gastric cancer, is characterized by hepatocellular carcinoma-like histology. As details of this disease remain unknown, the aim of this study was to evaluate the clinicopathological features of GHA.

Methods: $\quad$ From January 2001 to December 2010, 4563 patients were diagnosed with primary gastric cancer at Chang Gung Memorial Hospital, Linkou Medical Center. Ten (0.22\%) of these patients were diagnosed with GHA. The clinicopathological characteristics of these patients were collected retrospectively.

Results: $\quad$ The median age at diagnosis was 65.5 years, and six patients $(60 \%)$ were male. Seven patients had lymph node metastasis and five had distant metastasis, with the liver as the most common site (four cases). Serum alpha-fetoprotein (AFP) levels were elevated in seven of eight patients (median: $359.2 \mathrm{ng} / \mathrm{ml}$; range: $4.3-6535.6 \mathrm{ng} / \mathrm{ml}$ ). Endoscopically, six tumors were classified as Borrmann's type III cancer with the appearance of fungating mass le-

\section{At a Glance Commentary \\ Scientific background of the subject}

Gastric hepatoid adenocarcinoma (GHA), a rare type of primary gastric cancer, is characterized by hepatocellular carcinoma-like histology. The aim of this study was to evaluate the clinicopathological features of GHA.

\section{What this study adds to the field}

GHA is a histopathologic subtype of gastric cancer associated with a poor outcome, particularly when elevated carcinoembryonic antigen levels or distant metastasis is identified at diagnosis. Chemotherapy may prolong overall survival in select cases, and further attention and clinical studies are needed for this disease. sion with a purple, berry-like surface. Of the five patients without distant metastasis, all received curative-intent surgery and four received adjuvant chemotherapy. Four patients with distant metastasis received either palliative operation or chemotherapy, and one patient received neither operation nor chemotherapy due to a poor performance status. The median survival time was 7.2 months (range: 0.7-131.8 months), and the 5-year survival rate was $20 \%$. There was survival benefit in the chemotherapy groups.

Conclusions: GHA is a rare subtype of gastric cancer which is prone to lymph node and liver metastasis. Most GHAs appear as Borrmann's type III fungating mass lesion with a purple, berry-like surface. Although the prognosis of advanced stage GHA is poor, chemotherapy might provide some benefit. (Biomed J 2015;38:65-69)

Key words: alpha-fetoprotein, carcinoembryonic antigen, chemotherapy, gastric cancer, gastric hepatoid adenocarcinoma

$\mathrm{H}$ epatoid carcinoma, a tumor that histologically mimics the appearance of hepatocellular carcinoma, is most frequently identified in the stomach, but is also found in the ovary, lung, pancreas, gallbladder, cervix, and thymus. ${ }^{[1-5]}$ Gastric hepatoid adenocarcinoma (GHA) is a rare variant of primary gastric neoplasm which contains distinctive foci of hepatocellular differentiation, composed of large, polygonal cells with abundant eosinophilic cytoplasm that usually presents with a high level of serum alpha-fetoprotein (AFP) and is associated with an unfavorable outcome. ${ }^{[6-9]}$ In addition

From the ${ }^{1}$ Department of Gastroenterology, Chang Gung Memorial Hospital at Linkou, Chang Gung University College of Medicine, Taoyuan, Taiwan; ${ }^{2}$ Department of Internal Medicine, Taiwan Adventist Hospital, Taipei, Taiwan; ${ }^{3}$ College of Medicine, Chang Gung University, Taoyuan, Taiwan

Received: Aug. 13, 2013; Accepted: Jan. 28, 2014

Correspondence to: Dr. Cheng-Tang Chiu, Department of Gastroenterology and Hepatology, Chang Gung Memorial Hospital at Linkou. 5, Fusing St, Gueishan, Taoyuan 333, Taiwan (ROC). Tel: 886-3-3281200 ext. 8102; Fax: 886-3-3272236; E-mail: ctchiu@ adm.cgmh.org.tw DOI: $10.4103 / 2319-4170.126860$ 
to confirmation by morphology, GHA could be confirmed by several immunohistochemical markers such as albumin, AFP, alpha-1 antitrypsin, and transferrin.

Observational findings indicate that GHA is a new cancer subtype with similarities to early lymph node or liver metastasis, with a poor prognosis. As there is limited information concerning GHA, the aim of this study was to evaluate the clinicopathological features of GHA.

\section{METHODS}

\section{Patients}

To determine the cases of GHA, we performed a retrospective search of the pathological and medical record database from a medical center in North Taiwan and found that 4563 patients presented with newly diagnosed primary gastric cancer from January 2001 to December 2010. Patients with proven primary hepatocellular carcinoma were excluded. Of the total number of patients, $10(0.22 \%)$ presented with histological hepatoid differentiation classified as GHA and their medical records and endoscopic findings were reviewed.

Staging was done according to the American Joint Committee on Cancer (AJCC) TNM Staging Classification for Carcinoma of the Stomach, $7^{\text {th }}$ edition. ${ }^{[10]}$ This retrospective study was approved by the institutional review board of the Chang Gung Memorial Hospital, Taoyuan, Taiwan.

\section{Specimen and tissue collection}

All included cases had either endoscopic biopsy or surgical specimens available for pathological study. Histological classification was based on the World Health Organization classification system, ${ }^{[1]}$ and Lauren's classification was applied for surgical pathology.

\section{Tumor markers}

Serum tumor marker levels were analyzed during initial diagnosis. Serum carbohydrate antigen 19-9 (CA19-9) levels (normal level: <37 U/ml) were analyzed using an Abbott AxSYM immunoassay system. Levels of AFP (normal level: $<15 \mathrm{ng} / \mathrm{ml}$ ) and serum carcinoembryonic antigen (CEA, normal level: $<5 \mathrm{ng} / \mathrm{ml}$ ) were analyzed using Abbott ARCHITECT AFP and CEA chemiluminescent microparticle immunoassays, respectively.

\section{Statistical analyses}

Quantitative data are represented as mean and standard deviation (SD) or median and range. Categorical data are presented as rates and proportions.

\section{RESULTS}

The incidence rate of GHA among newly diagnosed gastric cancers in our study was $0.22 \%$. Patient characteristics are summarized in Table 1. Among the 10 patients with pathologically proven GHA, 6 were men and 4 were women, and the median age at diagnosis was 65.5 years (range: $46-77$ years). The major presenting symptom was abdominal pain (nine patients) accompanied by gastrointestinal bleeding episodes (seven patients). Nine patients were anemic, and the mean hemoglobin level was $8.87 \pm 2.63 \mathrm{~g} / \mathrm{dl}$. Neither abdominal sonography nor computed tomography determined any clinical evidence of liver cirrhosis. Of the six patients evaluated for hepatitis B or hepatitis C viral infection, one patient presented with chronic hepatitis $\mathrm{C}$.

Serum AFP levels were elevated in seven of eight patients (median: $359.2 \mathrm{ng} / \mathrm{ml}$; range: $4.3-6535.6 \mathrm{ng} / \mathrm{ml}$ ). CEA levels were obtained from nine patients (median: $4.7 \mathrm{ng} / \mathrm{ml}$; range: $1.63-203.38 \mathrm{ng} / \mathrm{ml}$ ): Four patients had elevated CEA levels and five had normal levels. All the five cases without distant metastasis had normal CEA level and the median survival was 21.9 months (range: 0.9-131.8 months). All others with distant metastasis presented elevated CEA levels and the median survival was 4.0 months (range: 0.7-8.9 months). Serum CA19-9 levels were recorded in six patients and found to be within normal limits.

Esophagogastroduodenoscopy revealed that the primary tumors were located at the body or antrum (including angle) in nine patients and presented at the cardia in one patient [Table 1]. Six tumors were classified as Borrmann's type III with the appearance of fungating mass lesion with a purple, berry-like surface [Table 2].

Among the eight surgical specimens from primary tumors, six had microscopic findings of either vascular, lymphatic, or perineural invasion. The most common histopathologic classification according to Lauren's classification was the intestinal type, and Helicobacter pylori infection was detected in four cases. Immunohistochemistry of the pathological specimens [Figure 1] determined that four of five $(80 \%)$ patients had positive hepatocyte paraffin antigen 1 (Hep-Par 1) staining, three of three (100\%) had positive AE1/AE3 staining, and seven of nine (77.8\%) had positive AFP staining.

Nine patients had either lymphatic and/or distant metastasis at the time of first diagnosis. The most common metastatic sites were the lymph nodes and liver. Of the five patients classified as stage IV, one received neither chemotherapy nor surgery due to a poor performance status, one received palliative fluorouracil (5-FU)-based chemotherapy, and three received palliative gastrectomy for symptom relief. The five patients without distant metastasis received radical resection, and one died after 
Table 1: Patient characteristics

\begin{tabular}{|c|c|c|c|c|c|c|c|c|c|c|c|}
\hline Age/sex & Initial symptom & $\begin{array}{l}\mathrm{HBsAg} / \\
\text { anti-HCV }\end{array}$ & Cirrhosis & H.pylori & $\begin{array}{c}\text { AFP } \\
(\mathrm{ng} / \mathrm{ml})\end{array}$ & $\begin{array}{c}\text { CEA } \\
(\mathrm{ng} / \mathrm{ml})\end{array}$ & $\begin{array}{c}\text { CA19-9 } \\
\text { (U/ml) }\end{array}$ & Location & Staging & $\begin{array}{l}\text { Distant } \\
\text { metastasis }\end{array}$ & PS \\
\hline $63 / \mathrm{F}$ & Epigastralgia; melena & ND & - & + & ND & N/A & ND & Antrum & T3N3M1 (IV) & Liver & 1 \\
\hline 69/M & Epigastralgia; melena & ND & - & + & 201.5 & 2.4 & ND & Antrum & T3N2M0 (IIIA) & None & 1 \\
\hline $62 / \mathrm{M}$ & Epigastralgia; melena & ND & - & - & ND & 3.7 & ND & Lower body & T3N3aM0 (IIIB) & None & 1 \\
\hline $64 / \mathrm{F}$ & Epigastralgia; melena & $-1-$ & - & - & 1133.7 & 2.6 & 25.2 & Lower body & T2N0M0 (IB) & None & 2 \\
\hline 69/M & Epigastralgia, melena & $-1-$ & - & - & 179.1 & 203.4 & 6.29 & Antrum & T3N0M1 (IV) & Liver; lung & 2 \\
\hline $46 / \mathrm{F}$ & Abdominal pain, BWL, jaundice & $-1-$ & - & ND & 55.0 & 103.9 & $<2$ & Lower body & T3N2M1 (IV) & Peritoneum & 3 \\
\hline $46 / \mathrm{M}$ & Abdominal pain & ND & - & + & 516.9 & 1.6 & ND & Angle & T1bN1M0 (IB) & None & 1 \\
\hline $69 / \mathrm{F}$ & Epigastralgia, melena & $-1-$ & - & ND & 6535.7 & 5.7 & $<2$ & Lower body & T3N0M1 (IV) & Liver & 2 \\
\hline 77/M & Epigastralgia, melena & $-/-$ & - & + & 3124.9 & 8.8 & $<2$ & Angle & T2bN1M1 (IV) & Liver & 2 \\
\hline 67/M & Hiccup, BWL & $-/+$ & - & - & 4.3 & 4.7 & $<2$ & Cardia & T2N2M0 (IIB) & None & 1 \\
\hline
\end{tabular}

Abbreviations: BWL: Body weight loss; F: Female; M: Male; ND: Not done; HBsAg: Hepatitis B antigen; Anti-HCV: Hepatitis C antibody; $H$. pylori: Helicobacter pylori infection; AFP: Alpha-fetoprotein; CEA: Carcinoembryonic antigen; CA19-9: Carbohydrate antigen 19-9; PS: Zubrod performance scale

Table 2: Pathological characteristics, treatment, and outcomes of the patients

\begin{tabular}{lllllll}
\hline Borrmann's classification & Lauren's classification & Differentiation & Microinvasion & Surgery & Chemotherapy & Outcome (months) \\
\hline III & Intestinal & Poor & Lymphatic & Palliative & None & Expired (2.6) \\
I & Intestinal & Poor & None & Radical & Adjuvant & Alive (131.8) \\
III & Intestinal & Differentiated & Lymphatic & Radical & Adjuvant & Expired (21.9) \\
III & Mixed & Poor & Perineural & Radical & Adjuvant & Expired (21.7) \\
III & Intestinal & Differentiated & Vascular & Palliative & None & Expired (2.6) \\
I & ND & Poor & ND & ND & None & Expired (0.7) \\
III & Intestinal & Poor & Lymphatic & Radical & Adjuvant & Alive (71.6) \\
II & ND & Poor & ND & ND & Palliative & Expired (8.9) \\
III & Intestinal & Poor & Vascular, lymphatic & Palliative & None & Expired (5.4) \\
I & Intestinal & Poor & None & Radical & None & Expired (0.9) \\
\hline
\end{tabular}

Abbreviation: ND: Not done

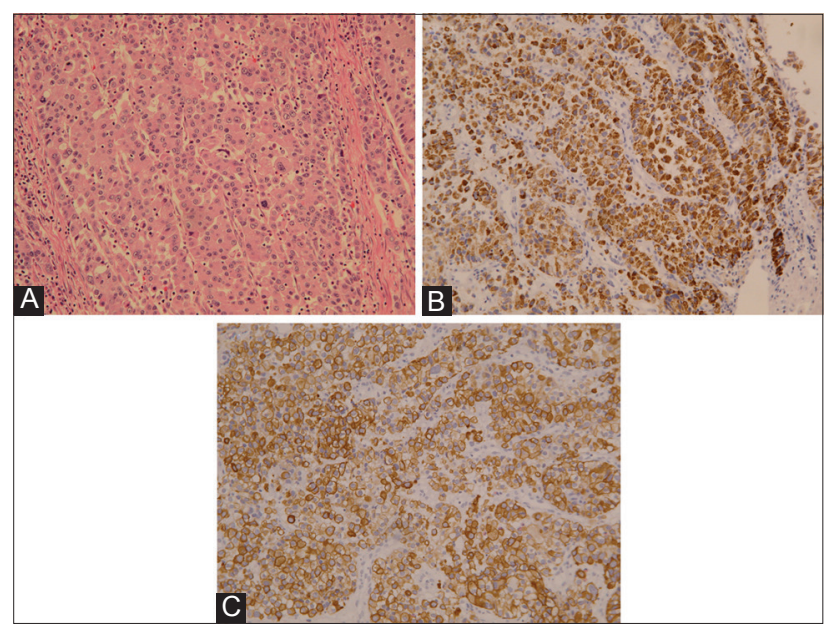

Figure 1: Microscopic features of gastric hepatoid adenocarcinoma: (A) Tumor cells with eosinophilic cytoplasm proliferating in a trabecular pattern; (B) Positive immunostain for Hep-Par 1 in a primary lesion; (C) Positive immunostain for AE1/AE3 in a primary lesion.

acquiring a nosocomial infection. The other four patients received 5-FU-based adjuvant chemotherapy. The median survival time from first diagnosis was 7.2 (range: 0.7-131.8) months, and the 5-year survival rate was $20 \%$. The medial survival of patients with GHA undergoing chemotherapy was 21.9 months (range: 8.9-131.8 months) with a 5-year survival rate of $40 \%$.

\section{DISCUSSION}

GHA is a rare disease with a reported incidence of $0.17-1 \%{ }^{[12,13]}$ Our study revealed a similar incidence rate $(0.22 \%)$ with a high incidence of metastasis. According to a previously published report, ${ }^{[14]}$ patients with GHA have an average age of 63.5 years, are predominantly male (2.32:1), have tumors mainly located at the antrum, and tend to have high levels of AFP. The most common initial presentation symptoms of GHA in our study included epigastralgia and anemia. Most patients (87.1\%) had either lymph node or distant metastasis. In our study, metastasis was predominantly seen in men in seventh decades and was always located at the distal part of gastric lumen. The most common presentation symptoms were abdominal pain and melena.

Previous studies showed that CEA levels were associated with the presence of distant metastasis and CA19-9 levels were related to nodal involvement. Elevated levels of each tumor marker were also associated with a worse 
prognosis for ordinary gastric adenocarcinoma. ${ }^{[15,16]}$ In our study, we found that elevated CEA levels were associated with poor prognosis in GHA, which was seldom surveyed in previous studies. As the CA19-9 levels of our patients were all within the normal range, the clinical significance between tumor markers and GHA requires further study.

Compared with ordinary, poorly differentiated gastric adenocarcinoma and hepatoma, blood vessel and lymphatic invasion, as well as evidence of metastasis, were found more frequently in $\mathrm{GHA}^{[17,18]}$ and may contribute to the poor prognosis. Chang et al. reported initial staging as an independent risk factor for GHA ${ }^{\left[{ }^{[19]}\right.}$ In our study, $90 \%$ of patients had either lymphatic and/or distant metastasis at the time of diagnosis, and these patients had worse overall survival. Therefore, as GHA is a rapidly progressing disease, better prognosis may be achieved by the early diagnosis of GHA.

Both palliative and adjuvant chemotherapy convey a survival benefit in ordinary gastric cancer ${ }^{[20,21]}$ and may be associated with the prognosis of GHA. ${ }^{[19]}$ Our data revealed that 5-FU-based chemotherapy might have some survival benefit.

The endoscopic findings of GHA were not discussed in previous studies. As presented in our data, Borrmann's type III was the most common presentation of GHA, ${ }^{[13]}$ which appeared as a fungating mass with a purple, berry-like surface [Figure 2]. Ulceration in the presence of recent hemorrhage was also observed in some cases. Clinically, patients in our study tended to be anemic and experienced melena, which was consisted with the endoscopic findings.

Two histological types of gastric adenocarcinoma, intestinal and diffuse, are recognized by Lauren's classification, and several studies have attempted to clarify the relationship between clinical outcome and histopathologic classification. In our study, most cases belonged to the intestinal type. In economically developed countries, the decreasing incidence of gastric cancer, especially intestinal type, is suggested to be related to environmental factors, such as $H$. pylori infection. ${ }^{[22-24]}$ However, data concerning both hepatoid adenocarcinoma and $H$. pylori infection were unavailable before this study. In this study, four surgical specimens were positive for $H$. pylori.

Hep-Par 1 is a marker considered specific for normal and neoplastic hepatocytes ${ }^{[25]}$ and is used to differentiate hepatocellular carcinoma and hepatoblastomas from cholangiocarcinomas and metastatic tumors to the liver ${ }^{[26]}$ However, in patients with primary gastric tumors without evidence of liver metastasis that were positive for Hep-Par 1 staining, primary GHA could not be ruled out, ${ }^{[27]}$ as Hep-Par 1 expression in extrahepatic cancers with hepatoid differentiation is quite variable. ${ }^{[28]}$ In our case series, Hep-Par 1 staining was useful, but not specific for differential diagnosis. Cases of ordinary gastric cancer with positive Hep-Par 1 staining

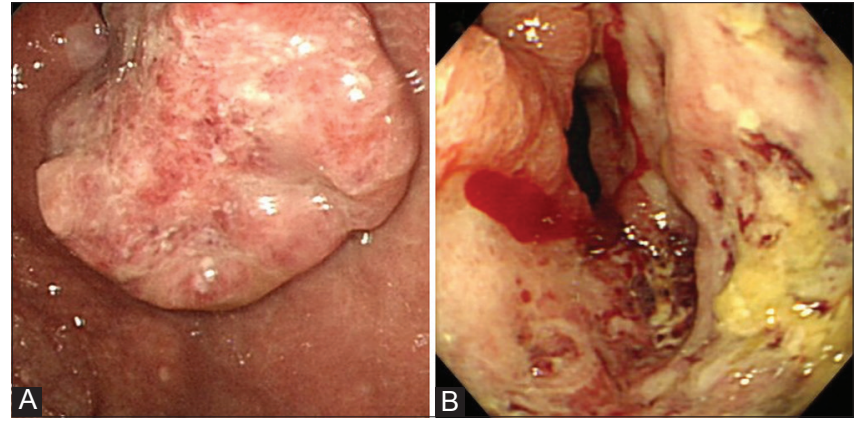

Figure 2: Endoscopic presentation of gastric hepatoid adenocarcinoma: (A) Fungating mass with purple, berry-like surface; (B) Ulceration in the presence of recent hemorrhage.

reported a better survival outcome than did cases that were negative for Hep-Par $1 ;{ }^{[29]}$ however, the limited number of cases in our study could not determine a significant difference in survival based upon Hep-Par 1 presentation.

Our study has several limitations. As GHA is a disease with a low incidence rate, our sample size was small. Furthermore, the data was collected in a retrospective manner in a single medical center, which may produce selection bias.

In conclusion, GHA is a rare subtype of gastric cancer which is prone to lymph node and liver metastasis. Most GHA presented as Borrmann's type III fungating mass lesion with a purple, berry-like surface and elevated AFP level. Although the prognosis of advanced stage GHA is poor, chemotherapy might provide some benefit.

\section{REFERENCES}

1. Hayashi Y, Takanashi Y, Ohsawa H, Ishii H, Nakatani Y. Hepatoid adenocarcinoma in the lung. Lung Cancer 2002;38:211-4.

2. Franke A, Strobel P, Fackeldey V, Schafer R, Goller T, Becker HP, et al. Hepatoid thymic carcinoma: Report of a case. Am J Surg Pathol 2004;28:250-6.

3. Takeuchi K, Kitazawa S, Hamanishi S, Inagaki M, Murata K. A case of alpha-fetoprotein-producing adenocarcinoma of the endometrium with a hepatoid component as a potential source for alpha-fetoprotein in a postmenopausal woman. Int J Gynecol Cancer 2006;16:1442-5.

4. Gakiopoulou H, Givalos N, Liapis G, Agrogiannis G, Patsouris E, Delladetsima I. Hepatoid adenocarcinoma of the gallbladder. Dig Dis Sci 2007;52:3358-62.

5. Huang SC, Chang HC, Yeh TS, Ng KF, Chen TC. Hepatoid microcarcinoma of the pancreas: A case report and review of the literature. Chang Gung Med J 2012;35:285-91.

6. Ishikura H, Kirimoto K, Shamoto M, Miyamoto Y, Yamagiwa H, Itoh $\mathrm{T}$, et al. Hepatoid adenocarcinomas of the stomach. An analysis of seven cases. Cancer 1986;58:119-26.

7. deLorimier A, Park F, Aranha GV, Reyes C. Hepatoid carcinoma of the stomach. Cancer 1993;71:293-6.

8. Nagai E, Ueyama T, Yao T, Tsuneyoshi M. Hepatoid adenocarcinoma of the stomach. A clinicopathologic and immunohistochemical analysis. Cancer 1993;72:1827-35. 
9. Ishikura H, Fukasawa Y, Ogasawara K, Natori T, Tsukada Y, Aizawa M. An AFP-producing gastric carcinoma with features of hepatic differentiation. A case report. Cancer 1985;56:840-8.

10. Washington $\mathrm{K} .7^{\text {th }}$ edition of the AJCC cancer staging manual: Stomach. Ann Surg Oncol 2010;17:3077-9.

11. Flejou JF. WHO Classification of digestive tumors: The fourth edition. Ann Pathol 2011;31 Suppl 5:S27-31.

12. Liu X, Cheng Y, Sheng W, Lu H, Xu X, Xu Y, et al. Analysis of clinicopathologic features and prognostic factors in hepatoid adenocarcinoma of the stomach. Am J Surg Pathol 2010;34:1465-71.

13. Baek SK, Han SW, Oh DY, Im SA, Kim TY, Bang YJ. Clinicopathologic characteristics and treatment outcomes of hepatoid adenocarcinoma of the stomach, a rare but unique subtype of gastric cancer. BMC Gastroenterol 2011;11:56.

14. Inagawa S, Shimazaki J, Hori M, Yoshimi F, Adachi S, Kawamoto T, et al. Hepatoid adenocarcinoma of the stomach. Gastric Cancer 2001;4:43-52.

15. Marrelli D, Roviello F, De Stefano A, Farnetani M, Garosi L, Messano A, et al. Prognostic significance of CEA, CA 19-9 and CA 72-4 preoperative serum levels in gastric carcinoma. Oncology 1999;57:55-62.

16. Kochi M, Fujii M, Kanamori N, Kaiga T, Kawakami T, Aizaki K, et al. Evaluation of serum CEA and CA19-9 levels as prognostic factors in patients with gastric cancer. Gastric Cancer 2000;3:177-86.

17. Gao YB, Zhang DF, Jin XL, Xiao JC. Preliminary study on the clinical and pathological relevance of gastric hepatoid adenocarcinoma. J Dig Dis 2007;8:23-8.

18. Roberts CC, Colby TV, Batts KP. Carcinoma of the stomach with hepatocyte differentiation (hepatoid adenocarcinoma). Mayo Clin Proc 1997;72:1154-60.

19. Zhang JF, Shi SS, Shao YF, Zhang HZ. Clinicopathological and prognostic features of hepatoid adenocarcinoma of the stomach. Chin Med J (Engl) 2011;124:1470-6.

20. GASTRIC (Global Advanced/Adjuvant Stomach Tumor Research International Collaboration) Group, Paoletti X, Oba K,
Burzykowski T, Michiels S, Ohashi Y, Pignon JP, et al. Benefit of adjuvant chemotherapy for resectable gastric cancer: A meta-analysis. JAMA 2010;303:1729-37.

21. Wagner AD, Grothe W, Haerting J, Kleber G, Grothey A, Fleig WE. Chemotherapy in advanced gastric cancer: A systematic review and meta-analysis based on aggregate data. J Clin Oncol 2006;24:2903-9.

22. Lauren PA, Nevalainen TJ. Epidemiology of intestinal and diffuse types of gastric carcinoma. A time-trend study in Finland with comparison between studies from high- and low-risk areas. Cancer 1993;71:2926-33.

23. Nakajima S, Nishiyama Y, Yamaoka M, Yasuoka T, Cho E. Changes in the prevalence of Helicobacter pylori infection and gastrointestinal diseases in the past 17 years. J Gastroenterol Hepatol 2010;25 Suppl 1:S99-110.

24. Wroblewski LE, Peek RM Jr, Wilson KT. Helicobacter pylori and gastric cancer: Factors that modulate disease risk. Clin Microbiol Rev 2010;23:713-39.

25. Fasano M, Theise ND, Nalesnik M, Goswami S, Garcia de Davila MT, Finegold MJ, et al. Immunohistochemical evaluation of hepatoblastomas with use of the hepatocyte-specific marker, hepatocyte paraffin 1 , and the polyclonal anti-carcinoembryonic antigen. Mod Pathol 1998;11:934-8.

26. Leong AS, Sormunen RT, Tsui WM, Liew CT. Hep Par 1 and selected antibodies in the immunohistological distinction of hepatocellular carcinoma from cholangiocarcinoma, combined tumours and metastatic carcinoma. Histopathology 1998;33:318-24.

27. Maitra A, Murakata LA, Albores-Saavedra J. Immunoreactivity for hepatocyte paraffin 1 antibody in hepatoid adenocarcinomas of the gastrointestinal tract. Am J Clin Pathol 2001;115:689-94.

28. Su JS, Chen YT, Wang RC, Wu CY, Lee SW, Lee TY. Clinicopathological characteristics in the differential diagnosis of hepatoid adenocarcinoma: A literature review. World J Gastroenterol 2013;19:321-7.

29. Fan Z, Li J, Dong B, Huang X. Expression of Cdx2 and hepatocyte antigen in gastric carcinoma: Correlation with histologic type and implications for prognosis. Clin Cancer Res 2005;11:6162-70. 